TRANSACTIONS OF THE

AMERICAN MATHEMATICAL SOCIETY

Volume 353, Number 5, Pages 1755-1766

S 0002-9947(01)02638-1

Article electronically published on January 16, 2001

\title{
PFAFFIAN SYSTEMS WITH DERIVED LENGTH ONE. THE CLASS OF FLAG SYSTEMS
}

\author{
MARÍA A. CAÑADAS-PINEDO AND CEFERINO RUIZ
}

\begin{abstract}
The incidence relations between a Pfaffian system and the characteristic system of its first derived system lead to obtain a characterization of Pfaffian systems with derived length one. Also, for flag systems, several properties are studied. In particular, an intrinsic proof of a result which determines the class of a system and of all the derived systems is given.
\end{abstract}

\section{INTRODUCTION}

In this paper, by means of a structure tensor and the incidence relations between a Pfaffian system $S$ and the characteristic system of the first derived system $S_{1}$, characterizations for the integrability of $S_{1}$ are obtained. In this paper we introduce new proof techniques which allow us to obtain the results in an instinsic way, not by using coordinates, as was traditional in earlier works concerning Pfaffian systems. So, for example, we give an intrinsic proof of a result that allows us to determine the class of a Pfaffian system and of its first derived system whenever the second derived system there exists and each of them has codimension one in the previous system. This result is particularly interesting for the flag case.

We have attempted to write this paper in a reasonably self-contained form. So, in Section 2, we recall the basic definitions and theorems from the theory of Pfaffian systems we will need.

In Section 3 we present a structure tensor defined by [8]. (We point out that we are not dealing with the well known Sternberg's structure tensor for $G$-structures.) This tensor $\mathbf{k}$ leads to a reformulation of Frobenius' theorem and it is used to define the characteristic and the derived systems of a Pfaffian system $S$.

This tensor $\mathbf{k}$, together with the study of the incidence between $S$ and the characteristic system of the first derived system, is used to obtain (Theorem 4.1) a characterization of the integrability of $S_{1}$. Also in Section 4, by using the same technique, other properties of Pfaffian systems are obtained.

In Section 5 the main goal is to characterize, in terms of the class, the flag systems (Theorem 5.4 and Corollary [5.3). However, we obtain results which can be applied in a more general situation.

We are interested in emphasizing that the technique used allows us to give direct and intrinsic proofs. Thus, for example, the necessary condition in Theorem 5.4

Received by the editors August 3, 1999 and, in revised form, October 1, 1999.

2000 Mathematics Subject Classification. Primary 58A17; Scondary 53C10.

Key words and phrases. Pfaffian systems, structure tensor, characteristic system, derived system, flag systems.

Research partially supported by a DGICYT grant PB94-0796.

(C)2001 American Mathematical Society 
was proved by Kumpera-Ruiz 8] and Gardner [5] independently, but the proof we give is not by using coordinates.

Likewise, we think it is interesting to compare the results obtained in Section 5 with some results that appear in [5]. By means of an intrinsic proof we obtain, in Corollary 5.2 the same conclusion from a different hypothesis. Also, we improve the necessary condition in Theorem 5.4 because we do not need to make any assumption about the integrability of $S_{2}$.

In Sections 4 and 5 several examples and counterexamples are shown in order to prove that, in most cases, it is not possible to improve the result. We also present an open problem in Section 4.

\section{Pfaffian Systems}

Our purpose in this section is to recall some basic definitions and results from the theory of Pfaffian systems. We refer the reader to [1] and [8] for the proofs unless otherwise indicated.

Let $M$ be a differentiable manifold of finite dimension $n$ which we will assume to be connected and of class $C^{\infty}$. We will denote by $T$ and $T^{*}$ the tangent and the cotangent bundle of $M$ respectively. Let $\Gamma(E)$ denote the module of the global sections of a vector bundle $E$ over $M$, and let $\Gamma_{\ell}(E)$ be the presheaf of the local sections of $E$.

Definition 2.1. A Pfaffian system on $M$ is a vector subbundle locally trivial $S$ of $T^{*}$.

Thus, if $S$ has rank $p$, locally $S$ is generated by linearly independent $p$ Pfaffian forms

$$
\omega^{1}, \ldots, \omega^{p} \in \Gamma_{\ell}(S)
$$

We shall represent

$$
S=\operatorname{span}\left\{\omega^{1}, \ldots, \omega^{p}\right\}
$$

omitting both the point and the neighborhood unless necessary to avoid misunderstandings.

Definition 2.2. Given a Pfaffian system $S$, an integral manifold of $S$ is a submanifold $\imath: N \hookrightarrow M$ such that $\imath^{*}(S)=0$.

Necessarily $\operatorname{dim}(N) \leq \operatorname{dim}(M)-\operatorname{rank}(S)$.

Definition 2.3. A Pfaffian system $S$ of rank $p$ is called completely integrable if, through each point of $M$, where $S$ is defined, there passes an $(n-p)$-dimensional integral manifold of $S$.

Definition 2.4. A maximal integral manifold of a Pfaffian system $S$ on $M$ is a connected integral manifold of a maximal dimension of $S$ whose image in $M$ is not a proper subset of any other connected integral manifold.

Theorem 2.1. Let $S$ be a completely integrable Pfaffian system on $M$. Then through each point $x$ in $M$ there passes a unique maximal integral manifold of $S$, and every connected integral manifold of $S$ through $x$ is contained in the maximal one. 
Given a Pfaffian system $S$, its annihilator $\Sigma=S^{\perp}$ is a subbundle of $T$, that is, a differentiable distribution. We will identify

$$
(T / \Sigma)^{*} \equiv S \quad\left(T^{*} / S\right) \equiv \Sigma^{*}
$$

Moreover, $\Sigma$ is completely integrable if and only if $S$ is completely integrable and $\Sigma$ and $S$ have the same integral manifolds.

The integrability of $S$ (or the one of the associated distribution) is characterized by Frobenius' theorem, whose classical version for Pfaffian systems can be stated in the following terms:

Theorem 2.2 (Frobenius). Let $S$ be a Pfaffian system generated by linearly independent 1 -forms $\omega^{1}, \ldots, \omega^{p}$. Then $S$ is completely integrable if and only if $d \omega^{i} \wedge$ $\omega^{1} \wedge \ldots \wedge \omega^{p}=0,1 \leq i \leq p$.

If Frobenius' theorem is satisfied, then, in a neighborhood, there exist local coordinates

$$
\left(x^{1}, \ldots, x^{n}\right)
$$

such that $S$ is locally generated by

$$
d x^{1}, \ldots, d x^{p} .
$$

This is the standard flat model for the Pfaffian system. A set of functions

$$
\left\{x^{1}, \ldots, x^{p}\right\}
$$

as above, is called a complete set of local first integrals of the completely integrable system $S$. The maximal integral manifolds, in this neighborhood, are given by

$$
\left\{x^{1}=c^{1}, \ldots, x^{p}=c^{p}\right\}
$$

where $c^{1}, \ldots, c^{p}$ are real constants depending on the point.

Definition 2.5. An automorphism or a symmetry of $S$ (also of $\Sigma$ ) is a diffeomorphism $\varphi$ of $M$ such that $\varphi^{*}(S)=S$ (or $\varphi_{*}(\Sigma)=\Sigma$ ). We shall denote $\mathcal{A} u t(S)$ the group of automorphisms of $S$.

Definition 2.6. An infinitesimal automorphism, or an infinitesimal symmetry, of $S$ (also of $\Sigma$ ) is a vector field which generates a local 1-parameter group of automorphisms of $S$. We will denote $L(S)$ the set of all the infinitesimal automorphisms of $S$.

It is easily checked that $\xi \in L(S)$ if and only if

$$
L_{\xi}\left(\Gamma_{\ell}(S)\right) \subset \Gamma_{\ell}(S) \quad\left(\text { or } L_{\xi}\left(\Gamma_{\ell}(\Sigma)\right) \subset \Gamma_{\ell}(\Sigma)\right)
$$

where $L_{\xi}$ is the Lie derivative with respect to $\xi$.

Definition 2.7. An infinitesimal automorphism $\xi \in L(S)$ is called characteristic if it is tangent to $\Sigma$, that is, if $\xi \in \Gamma_{\ell}(\Sigma)$.

The module

$$
L_{c}(S)=L(S) \cap \Gamma_{\ell}(\Sigma)
$$

of the characteristic vector fields induces a distribution, which might be singular, and which we shall denote by $\Delta=\Delta(S)$ : in each point $x$ of $M, \Delta_{x}$ is the subspace of $T_{x} M$ generated by the values in $x$ of the characteristic vector fields.

Since $\varphi \in L_{c}(S)$ if and only if

$$
i_{\varphi} \omega=i_{\varphi} i_{\eta} d \omega=0
$$


for every $\omega \in \Gamma_{\ell}(S)$ and every $\eta \in \Gamma_{\ell}(\Sigma)$, the set of the vector fields of $M$ which take value in $\Delta$ is $L_{c}(S)$.

Whenever the dimension of $\Delta_{x}$ is constant, that is, whenever $\Delta$ is a distribution, it is called, following Élie Cartan, the characteristic distribution of $S$ and its annihilator $C=C(S) \subset T^{*}$ is the characteristic system of $S$. In this case, the integer $\operatorname{rank}(C(S))$ is named the class of the Pfaffian system $S$ and it is denoted by $\operatorname{class}(S)$.

Henceforth, we shall assume that $S$ is a Pfaffian system with constant class, that is, $\operatorname{dim}\left(\Delta_{x}\right)$ is constant.

Theorem 2.3. The characteristic system $C$ of a Pfaffian system $S$ is always a completely integrable system.

Proposition 2.1. Let $S$ be a Pfaffian system. The following are equivalent:

(a) $S$ is completely integrable.

(b) $\Delta=\Sigma$.

(c) $C=S$.

Theorem 2.4. Let $S$ be a Pfaffian system. Then the characteristic system of $S$ is the smallest completely integrable Pfaffian system with the property that if $\left\{x^{1}, \ldots, x^{p}\right\}$ is a local system of first integrals, then locally there exist generators of $S$ which only depend on $\left\{x^{1}, \ldots, x^{p}\right\}$ and their differentials.

The class, therefore, is the minimum number of variables necessary in order to write down local generators of the system. Those variables are called the characteristic variables of the system $S$.

\section{Structure tensor. Derived systems}

The structure tensor of a Pfaffian system $S$ ([8]) is defined as the vector bundle morphism

$$
\mathbf{k}: \Sigma \otimes S \longrightarrow \Sigma^{*} \equiv T^{*} / S
$$

given by

$$
\mathbf{k}(\xi \otimes \omega)=\left(\mathbf{q} \circ L_{\xi}\right)(\omega)
$$

for all $\xi \in \Gamma(\Sigma)$ and $\omega \in \Gamma(S)$, where

$$
\mathbf{q}: T^{*} \longrightarrow T^{*} / S
$$

is the quotient morphism.

Notice that $\mathbf{k}(\xi \otimes \omega)=\mathbf{q}\left(i_{\xi}(d \omega)\right)$.

Proposition 3.1. The rank of $\mathbf{k}$ is constant if and only if the dimension of $\Delta_{x}$ is constant.

In this case, we establish:

Theorem 3.1. Let $S$ be a Pfaffian system of constant class, then

$$
C(S)=\boldsymbol{q}^{-1}(\mathbf{k}(\Sigma \otimes S))
$$

and

$$
\operatorname{class}(S)=\operatorname{rank}(\mathbf{k})+\operatorname{rank}(S)
$$

We can formulate Frobenius' theorem by means of the structure tensor in the following terms: 
Theorem 3.2. A Pfaffian system $S$ is completely integrable if and only if $\mathbf{k} \equiv 0$.

Remark 3.1. Theorem 3.2 allows us to consider the structure tensor of a Pfaffian system as a curvature for the flatness (integrability) of a Pfaffian system.

Proposition 3.2. The rank of $\mathbf{k}$ is always different from 1; as a consequence, the class of a Pfaffian system $S$ is either equal to its rank (if $S$ is completely integrable) or strictly greater than rank $(S)+1$ (if $S$ is not completely integrable).

Definition 3.1. In each point $x \in M,\left(S_{1}\right)_{x}$ is defined as the right kernel of the tensor $\mathbf{k}_{x}$, i.e.,

$$
\left(S_{1}\right)_{x}=\left\{\omega \in S_{x} / \mathbf{k}(\xi \otimes \omega)=0, \forall \xi \in \Sigma_{x}\right\} .
$$

Whenever the dimension of these spaces is constant, the Pfaffian system $S_{1}$ obtained is called, following Élie Cartan, the first derived system of $S$.

Notice that, since

$$
d \omega(\xi, \eta)=-\omega([\xi, \eta]), \forall \omega \in \Gamma_{\ell}(S), \xi, \eta \in \Gamma_{\ell}(\Sigma)
$$

the field $\Sigma_{1}=S_{1}^{\perp}$, consisting of the annihilators of $S_{1}$, is the distribution induced by the submodule of $\Gamma_{\ell}(T)$ generated by

$$
\Gamma_{\ell}(\Sigma) \cup\left[\Gamma_{\ell}(\Sigma), \Gamma_{\ell}(\Sigma)\right]
$$

Theorem 3.3. A Pfaffian system $S$ is completely integrable if and only if $S=S_{1}$.

Since $S_{1}$ is a subbundle of $S$ canonically associated to $S$, we have

\section{Theorem 3.4.}

$$
\begin{array}{rlrl}
\mathcal{A} u t(S) & \subset \mathcal{A} u t\left(S_{1}\right), & L(S) & \subset L\left(S_{1}\right), \\
L_{c}(S) & \subset L_{c}\left(S_{1}\right), & \Delta(S) \subset \Delta\left(S_{1}\right) .
\end{array}
$$

We shall denote $\Delta\left(S_{1}\right)=\Delta_{1}, C\left(S_{1}\right)=C_{1}$ and $\mathbf{k}_{1}$ the structure tensor of $S_{1}$.

The successive derived systems are defined inductively by

$$
S_{\mathrm{r}+1}=\left(S_{\mathrm{r}}\right)_{1}
$$

Whenever all the derived systems exist we obtain a decreasing sequence of Pfaffian systems,

$$
S=S_{0} \supseteq S_{1} \supseteq \ldots \supseteq S_{\mathrm{r}} \supseteq S_{\mathrm{r}+1} \supseteq \ldots
$$

whose last term is a completely integrable Pfaffian system (which might be trivial).

Definition 3.2. A Pfaffian system which satisfies the above condition is called totally regular. The derived length of a totally regular Pfaffian system $S$ is the smallest nonnegative integer $\ell$ such that $S_{\ell}=S_{\ell+1}$.

For the $\mathrm{r}^{\text {th }}$ derived system, $S_{\mathrm{r}}$ of $S, \mathbf{k}_{\mathrm{r}}$ represents its structure tensor and we denote

$$
\Delta_{\mathrm{r}}=\Delta\left(S_{\mathrm{r}}\right), C_{\mathrm{r}}=C\left(S_{\mathrm{r}}\right)
$$

Theorem 3.5.

$$
\mathbf{k}_{1}\left(\Sigma \otimes S_{1}\right) \subseteq S / S_{1}
$$

Proof. Since the diagram 


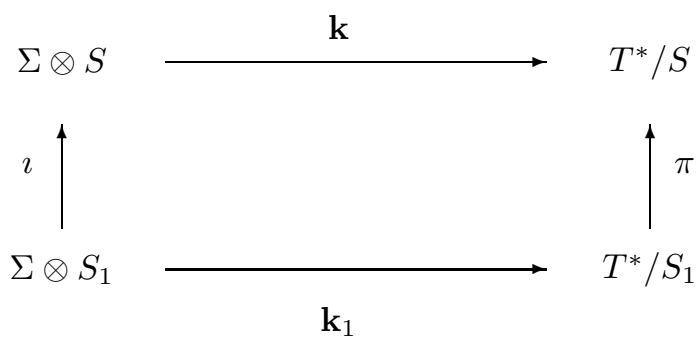

is commutative and the sequence

$$
0 \longrightarrow S / S_{1} \longrightarrow T^{*} / S_{1} \longrightarrow T^{*} / S \longrightarrow 0
$$

is exact, condition

$$
\mathbf{k}\left(\Sigma \otimes S_{1}\right)=\{0\}
$$

implies

$$
\mathbf{k}_{1}\left(\Sigma \otimes S_{1}\right) \subseteq S / S_{1}
$$

This property, which is essential in this work, allows us, for example, to define, for a totally regular Pfaffian system with derived length $\ell$, the reduced tensor by

$$
\mathbf{k}_{(\mathrm{r})}: \Sigma \otimes S_{\mathrm{r}} \longrightarrow S_{\mathrm{r}-1} / S_{\mathrm{r}}, \quad 1 \leq \mathrm{r} \leq \ell,
$$

as the restriction of the structure tensor

$$
\mathbf{k}_{\mathrm{r}}: \Sigma_{\mathrm{r}} \otimes S_{\mathrm{r}} \longrightarrow T^{*} / S_{\mathrm{r}}
$$

and $\mathbf{k}_{(0)}=\mathbf{k}$.

Since $S_{\mathrm{N}}(\mathrm{N} \geq \ell)$ is completely integrable, $\mathbf{k}_{\mathrm{N}}=\mathbf{k}_{(\mathrm{N})}=0$ for every integer $\mathrm{N} \geq \ell$

\section{ThE CHARACTERISTIC SYSTEM OF THE FIRST DERIVED SYSTEM}

In this section the goal is to prove some results concerning the incidence relations between a Pfaffian system $S$ and the characteristic system of its first derived system $S_{1}$. These relations allow us to characterize the integrability of $S_{1}$, that is, we characterize the totally regular Pfaffian systems with derived length one.

Theorem 4.1. Let $S$ be a totally regular Pfaffian system with derived length $\ell$. The following conditions are equivalent:

(a) $\ell=1$, that is, $S_{1}$ is completely integrable.

(b) $C_{1} \cap S=S_{1}$.

(c) $\mathbf{k}_{1}\left(\Sigma \otimes S_{1}\right)=\{0\}$.

(d) $C_{1} \cap S=C_{1}$.

(e) $\mathbf{k}_{1}\left(\Sigma \otimes S_{1}\right)=C_{1} / S_{1}$.

(f) For every complement $\widehat{\Sigma}$ of $\Sigma$ in $\Sigma_{1}, \mathbf{k}_{1}\left(\widehat{\Sigma} \otimes S_{1}\right)=\{0\}$.

(g) There exists a complement $\widehat{\Sigma}$ of $\Sigma$ in $\Sigma_{1}$ such that $\mathbf{k}_{1}\left(\widehat{\Sigma} \otimes S_{1}\right)=\{0\}$.

Proof. $S_{1}$ is completely integrable iff $\mathbf{k}_{1} \equiv 0$ iff $C_{1}=S_{1}$. Therefore, if we suppose that $S_{1}$ is completely integrable, we have

$$
C_{1} \cap S_{1}=S_{1} \cap S=S_{1}=C_{1}, \quad \mathbf{k}_{1} \equiv 0, \quad C_{1} / S_{1}=\{0\}
$$


and, as a consequence, conditions (b) through (g) are necessary for $S_{1}$ to be completely integrable.

Let us suppose $C_{1} \cap S=S_{1}$. Since

$$
\mathbf{k}_{1}\left(\Sigma \otimes S_{1}\right) \subseteq S / S_{1} \quad \text { and } \quad \mathbf{k}_{1}\left(\Sigma_{1} \otimes S_{1}\right)=C_{1} / S_{1},
$$

it follows that

$$
\mathbf{k}_{1}\left(\Sigma \otimes S_{1}\right) \subseteq\left(C_{1} \cap S\right) / S_{1}=\{0\}
$$

that is, we get $(\mathrm{c})$.

If $\mathbf{k}_{1}\left(\Sigma \otimes S_{1}\right)=\{0\}$ then the local sections of $\Sigma$ are infinitesimal automorphisms of $S_{1}$, i.e., $\Sigma \subseteq \Delta_{1}$, or, equivalently, $C_{1} \subseteq S$ and (d) holds.

Moreover, $C_{1}$ is a proper subset of $S$ if $S$ is not completely integrable because $C_{1}$ is always a completely integrable system.

From (d), as $C_{1} \cap S=C_{1}$ is equivalent to $C_{1} \subseteq S$, we have

$$
S_{1} \subseteq C_{1} \subseteq S
$$

Since $C_{1}$ is completely integrable and $\Sigma \subseteq \Delta_{1}$, we obtain

$$
\mathbf{k}\left(\Sigma \otimes C_{1}\right)=\{0\} .
$$

This means that $C_{1}$ is included in the right kernel of $\mathbf{k}$, which is $S_{1}$. Therefore, $C_{1}=S_{1}$ is completely integrable, and so the first four conditions are equivalent.

Condition (e) is also equivalent to the ones above because, if

$$
\mathbf{k}_{1}\left(\Sigma \otimes S_{1}\right)=C_{1} / S_{1},
$$

then $C_{1} \subseteq S$, i.e., $C_{1} \cap S=C_{1}$ and thus (e) implies (d).

Finally, from (g) if, for a complement $\widehat{\Sigma}$ of $\Sigma$ in $\Sigma_{1}$,

$$
\mathbf{k}_{1}\left(\widehat{\Sigma} \otimes S_{1}\right)=\{0\}
$$

then

$$
\mathbf{k}_{1}\left(\Sigma \otimes S_{1}\right)=C_{1} / S_{1}
$$

that is, $S_{1}$ is completely integrable.

In terms of the reduced tensor $\mathbf{k}_{(1)}$ we can state conditions (c) and (e) in the following corollary:

Corollary 4.1. The following are equivalent:

i) $S_{1}$ is completely integrable (i.e., $\mathbf{k}_{1} \equiv 0$ ).

ii) $\mathbf{k}_{(1)} \equiv 0$.

iii) $\operatorname{Im}\left(\mathbf{k}_{1}\right)=\operatorname{Im}\left(\mathbf{k}_{(1)}\right)$ (i.e., $\mathbf{k}_{1}\left(\Sigma_{1} \otimes S_{1}\right)=\mathbf{k}_{(1)}\left(\Sigma \otimes S_{1}\right)$ ).

Proposition 4.1. If $\operatorname{rank}\left(\mathbf{k}_{(1)}\right)=\operatorname{dim}\left(S / S_{1}\right)=p$, then $S \subseteq C_{1}$.

Moreover, $S=C_{1}$ if and only if $p=0$.

Proof. Since $\mathbf{k}_{1}\left(\Sigma \otimes S_{1}\right) \subseteq S / S_{1}$, we have $\mathbf{k}_{1}\left(\Sigma \otimes S_{1}\right)=S / S_{1}$ and then $S \subseteq C_{1}$. Moreover, $p=0$ iff $S$ is completely integrable iff $S=S_{1}=C_{1}$.

As a consequence of Theorem 4.1 and Proposition 4.1 we have a new corollary:

Corollary 4.2. If $\operatorname{dim}\left(S / S_{1}\right)=1$, then or $S_{1}$ is completely integrable or $S \subsetneq C_{1}$.

Proof. As $0 \leq \operatorname{rank}\left(\mathbf{k}_{(1)}\right)=\operatorname{dim}\left(\mathbf{k}_{1}\left(\Sigma \otimes S_{1}\right)\right) \leq \operatorname{dim}\left(S / S_{1}\right)=1$, then

i) $\operatorname{dim}\left(\mathbf{k}_{1}\left(\Sigma \otimes S_{1}\right)\right)=0 \Longleftrightarrow S_{1}$ is completely integrable,

or

ii) $\operatorname{dim}\left(\mathbf{k}_{1}\left(\Sigma \otimes S_{1}\right)\right)=1=\operatorname{dim}\left(S / S_{1}\right) \Longrightarrow S \subsetneq C_{1}$. 
Remark 4.1. Example 4.1 shows that if the codimension of $S_{1}$ in $S$ is greater than one, the nonintegrability of $S_{1}$ does not imply $S \subset C_{1}$.

Example 4.1. In dimension 6, the Pfaffian system $S$ of rank 3, locally generated by the 1 -forms

$$
\begin{aligned}
& \omega^{1}=d x^{1}+x^{2} d x^{6} \\
& \omega^{2}=d x^{2}+x^{3} d x^{6} \\
& \omega^{3}=d x^{4}+x^{5} d x^{6}
\end{aligned}
$$

has as its first derived system $S_{1}$, the system of constant rank 1 ,

$$
S_{1}=\operatorname{span}\left\{\omega^{1}\right\} \text {. }
$$

In this case $\omega^{3} \notin C_{1}=\operatorname{span}\left\{d x^{1}, d x^{2}, d x^{6}\right\}$ and thus $S \not \subset C_{1}$.

Proposition 4.2. If any of the next conditions hold:

(a) $S_{1}$ is completely integrable.

(b) $\operatorname{dim}\left(S / S_{1}\right)=1$.

Then $\operatorname{Im}\left(\mathbf{k}_{(1)}\right)=\left(C_{1} \cap S\right) / S_{1} \quad$ (i.e., $\left.\mathbf{k}_{1}\left(\Sigma \otimes S_{1}\right)=\left(C_{1} \cap S\right) / S_{1}\right)$.

Proof. For (a), it is enough to take into account that $\mathbf{k}_{1}=0$ and $C_{1} \cap S=S_{1}$.

For (b) as $\mathbf{k}_{1}\left(\Sigma \otimes S_{1}\right) \subseteq S / S_{1}$ and $\operatorname{dim}\left(S / S_{1}\right)=1$

or $\mathbf{k}_{1}\left(\Sigma \otimes S_{1}\right)=\{0\}$; that is, $S_{1}$ is completely integrable, or $\operatorname{dim} \mathbf{k}_{1}\left(\Sigma \otimes S_{1}\right)=1$, i.e., $\mathbf{k}_{1}\left(\Sigma \otimes S_{1}\right)=S / S_{1}$ and then $S / S_{1} \subset C_{1} / S_{1}$.

Thus $S / S_{1}=\left(C_{1} \cap S\right) / S_{1}$, i.e., $\mathbf{k}_{1}\left(\Sigma \otimes S_{1}\right)=\left(C_{1} \cap S\right) / S_{1}$.

Remark 4.2. Note that we have proved $\mathbf{k}_{1}\left(\Sigma \otimes S_{1}\right)=\left(C_{1} \cap S\right) / S_{1}$ unless $\operatorname{dim}\left(S / S_{1}\right)$ $>1$. In this case the problem is still open.

\section{Flag Pfaffian systems}

In this section the first step is to analyze those Pfaffian systems such that the codimension of its first derived system, $S_{1}$, in $S$ is one. Next we study the case $\operatorname{dim}\left(S / S_{1}\right)=\operatorname{dim}\left(S_{1} / S_{2}\right)=1$, which means that the second derived system verifies the same property in relation to the first one. Finally, we extend the results to totally regular systems with derived length $\ell$,

$$
S_{\ell} \subset \ldots \subset S_{1} \subset S_{0}=S
$$

such that, $\operatorname{dim}\left(S_{\mathrm{r}-1} / S_{\mathrm{r}}\right)=1$, for $1 \leq \mathrm{r} \leq \ell$, or equivalently,

$$
\operatorname{dim}\left(S / S_{\ell}\right)=\ell=\operatorname{rank}(S)-\operatorname{rank}\left(S_{\ell}\right) .
$$

Also, we obtain a sufficient condition for $S_{1}$ to have codimension one in $S$.

Definition 5.1. A totally regular Pfaffian system $S$ is named a flag (Pfaffian) system when its derived length $\ell$ is equal to $\operatorname{rank}(S)$.

These systems are called special systems by Goursat [5]. The most representative example is the contact system in the space of holonomic jets for functions of one variable.

As a first result we can apply Proposition 4.2 to flag systems:

Proposition 5.1. If $S$ is a flag Pfaffian system with derived length $\ell$, then

$$
\mathbf{k}_{\mathrm{i}}\left(\Sigma_{\mathrm{i}-1} \otimes S_{\mathrm{i}}\right)=\left(C_{\mathrm{i}} \cap S_{\mathrm{i}-1}\right) / S_{\mathrm{i}}
$$

for every $1 \leq \mathrm{i} \leq \ell$. 
Theorem 5.1. Let $S$ be a Pfaffian system with first derived system $S_{1}$. If $S \subset C_{1}$ and $\operatorname{rank}\left(\mathbf{k}_{1}\right)=2$, then $\operatorname{rank}(\mathbf{k})=2$.

Proof. As $\operatorname{rank}\left(\mathbf{k}_{1}\right)=2, S_{1}$ has codimension two in $C_{1}$. Since $S_{1} \subset S \subset C_{1}$, we deduce that $S$ has codimension one in $C_{1}$ or, equivalently, $\Delta_{1}$ has codimension one in $\Sigma$. So, if $\widehat{\Delta}_{1}$ is a complement of $\Delta_{1}$ in $\Sigma$, that is, $\Sigma=\Delta_{1} \oplus \widehat{\Delta}_{1}$, then $\operatorname{dim}\left(\widehat{\Delta}_{1}\right)=1$.

On the other hand, from

$$
\operatorname{dim}\left(C_{1} / S_{1}\right)=2 \text { and } S_{1} \subset S \subset C_{1}
$$

it follows that $\operatorname{dim}\left(S / S_{1}\right)=1$ and thus, if $S=S_{1} \oplus \widehat{S}_{1}, \widehat{S}_{1}$ has dimension one.

Therefore we can descompose

$$
\Sigma \otimes \widehat{S}_{1}=\left(\Delta_{1} \otimes \widehat{S}_{1}\right) \oplus\left(\widehat{\Delta}_{1} \otimes \widehat{S}_{1}\right)
$$

and as $\operatorname{dim}\left(\widehat{\Delta}_{1} \otimes \widehat{S}_{1}\right)=1$, we have

$$
\operatorname{dim}\left(\mathbf{k}\left(\widehat{\Delta}_{1} \otimes \widehat{S}_{1}\right)\right) \leq 1 .
$$

Also, since $C_{1}$ is completely integrable, the vector fields of $\Delta_{1}$ are characteristic vector fields of $C_{1}$, that is $L_{\Delta_{1}}\left(C_{1}\right) \subseteq C_{1}$ and therefore

$$
\mathbf{k}\left(\Delta_{1} \otimes \widehat{S}_{1}\right) \subseteq C_{1} / S .
$$

This implies

$$
\operatorname{dim}\left(\mathbf{k}\left(\Delta_{1} \otimes \widehat{S}_{1}\right)\right) \leq 1
$$

From (11) and (2),

$$
\operatorname{rank}(\mathbf{k}) \leq 2 .
$$

But $S$ is not a completely integrable system and so

$$
\operatorname{rank}(\mathbf{k}) \geq 2
$$

and therefore

$$
\operatorname{rank}(\mathbf{k})=2 .
$$

Notice that Gardner [5] obtains the same conclusion, that is, $\operatorname{rank}(\mathbf{k})=2$ by changing the hypothesis $S \subset C_{1}$ and $\operatorname{rank}\left(\mathbf{k}_{1}\right)=2$ by $\operatorname{dim}\left(S / S_{1}\right)=1$ and $\operatorname{rank}\left(\mathbf{k}_{1}\right) \neq 0$.

We can state the above theorem in terms of the class in the next corollary:

Corollary 5.1. If $S \subset C_{1}$ and class $\left(S_{1}\right)=\operatorname{rank}\left(S_{1}\right)+2$, then

$$
\operatorname{class}(S)=\operatorname{rank}(S)+2 .
$$

From the proof of Theorem 5.1 we obtain

Corollary 5.2. If $\operatorname{rank}\left(\mathbf{k}_{1}\right)=2$ and $S \subset C_{1}$, then the codimension of $S_{1}$ in $S$ is one.

Remark 5.1. Example 4.1 shows that we cannot deduce $\operatorname{rank}(\mathbf{k})=2$ from the condition $\operatorname{rank}\left(\mathbf{k}_{1}\right)=2$.

Remark 5.2. Below we give two examples which prove that, even in the flag case, if $\operatorname{rank}\left(\mathbf{k}_{1}\right) \neq 2$, in general, the rank of $\mathbf{k}$ cannot be determined. 
Example 5.1. Let us consider, in dimension six, the Pfaffian system of rank two, locally generated by

$$
\begin{aligned}
& \omega^{1}=d x^{1} \\
& \omega^{2}=d x^{2}+x^{3} d x^{4}+x^{5} d x^{6} .
\end{aligned}
$$

The derived system is a completely integrable system of rank one locally generated by $\omega^{1}$. So $\operatorname{rank}\left(\mathbf{k}_{1}\right)=0$ whereas $\operatorname{rank}(\mathbf{k})=4$, that is, the class of $S$ is six, the characteristic system of $S$ in every point is the whole cotangent space in the point.

Example 5.2. Let us consider, in dimension five, the Pfaffian system of rank three, locally generated by the 1 -forms:

$$
\begin{aligned}
\omega^{1} & =d x^{1}+\left(x^{3}+x^{4} x^{5}\right) d x^{4} \\
\omega^{2} & =d x^{2}+x^{3} d x^{5} \\
\omega^{3} & =d x^{3}+x^{4} d x^{5} .
\end{aligned}
$$

The derived system $S_{1}$ is generated by $\omega^{1}$ and $\omega^{2}$. This system determines completely, in dimension five, the system $S$, that is, given the system $S_{1}$, of rank two locally generated by $\omega^{1}$ and $\omega^{2}, S$ is the only Pfaffian system on this manifold which has $S_{1}$ as its first derived system.

The derived system of $S_{1}, S_{2}$, is trivial in this case and we have

$$
\operatorname{rank}(\mathbf{k})=2 \text { and } \operatorname{rank}\left(\mathbf{k}_{1}\right)=3 ;
$$

that is, in both cases, the characteristic system of $S$ in each point is the whole cotangent space in the point.

This situation is the one that É. Cartan [4] describes saying that there exists a univocal correspondence between $S$ and $S_{1}$ in dimension five (also see [3]).

Theorem 5.2. If $\operatorname{rank}(\mathbf{k})=2$, then $S_{1}$ has codimension one in $S$.

Proof. Since $\operatorname{rank}(\mathbf{k})=2, S$ has codimension two in $C$, or, equivalently, $\Delta$ has codimension two in $\Sigma$. Let $X_{1}$ and $X_{2}$ be linearly independent local generators of a complement of $\Delta$ in $\Sigma$. Let us call

$$
\widetilde{\Sigma}=\Sigma \oplus \operatorname{span}\left\{\left[X_{1}, X_{2}\right]\right\}
$$

and let $\widetilde{S}=\widetilde{\Sigma}^{\perp}$. As $\widetilde{S} \subset S$ and $\operatorname{dim}(S / \widetilde{S})=1$ it suffices to prove $\widetilde{S}=S_{1}$.

Let $\omega \in \Gamma_{\ell}(\widetilde{S})$. For $\mathrm{i}, \mathrm{j}=1,2$

$$
\left(L_{X_{\mathrm{i}}} \omega\right)\left(X_{\mathrm{j}}\right)=d \omega\left(X_{\mathrm{i}}, X_{\mathrm{j}}\right)=-\omega\left(\left[X_{\mathrm{i}}, X_{\mathrm{j}}\right]\right)=0 .
$$

For $\xi \in \Gamma_{\ell}(\Delta)$,

$$
\left(L_{X_{\mathrm{i}}} \omega\right)(\xi)=d \omega\left(X_{\mathrm{i}}, \xi\right)=-d \omega\left(\xi, X_{\mathrm{i}}\right)=-\left(L_{\xi} \omega\right)\left(X_{\mathrm{i}}\right)=0 .
$$

In addition to this, $\mathbf{k}(\Delta \otimes S)=\{0\}$. Thus $\mathbf{k}(\Delta \otimes \widetilde{S})=\{0\}$ and therefore $\widetilde{S} \subseteq S_{1}$. Since $S \neq S_{1}$, this implies $\widetilde{S}=S_{1}$.

Theorem 5.3. If $\operatorname{dim}\left(S / S_{1}\right)=1$ and $\operatorname{dim}\left(S_{1} / S_{2}\right)=1$, then $\operatorname{rank}\left(\mathbf{k}_{1}\right)=2$.

Therefore, the class of $S_{1}$ is constant and equal to $\operatorname{rank}\left(S_{1}\right)+2$.

Proof. As $\operatorname{dim}\left(S / S_{1}\right)=1, \Sigma_{1}=\widehat{\Sigma} \oplus \Sigma$ with $\operatorname{dim}(\widehat{\Sigma})=1$ and

$$
\mathbf{k}_{1}\left(\Sigma_{1} \otimes S_{1}\right)=\mathbf{k}_{1}\left(\widehat{\Sigma} \otimes S_{1}\right)+\mathbf{k}_{1}\left(\Sigma \otimes S_{1}\right) .
$$

Since $\mathbf{k}_{1}\left(\Sigma \otimes S_{1}\right) \subseteq S / S_{1}$, we have

$$
\operatorname{dim}\left(\mathbf{k}_{1}\left(\Sigma \otimes S_{1}\right)\right) \leq 1
$$


On the other hand, as $S_{2}$ is the first derived system of $S_{1}$,

$$
\mathbf{k}_{1}\left(\Sigma_{1} \otimes S_{2}\right)=\{0\}
$$

which implies, if $\widehat{S}_{2}$ is a complement of $S_{2}$ in $S_{1}$,

$$
\mathbf{k}_{1}\left(\widehat{\Sigma} \otimes S_{1}\right)=\mathbf{k}_{1}\left(\widehat{\Sigma} \otimes \widehat{S}_{2}\right) .
$$

So, since $\operatorname{dim}(\widehat{\Sigma})=1$ and $\operatorname{dim}\left(\widehat{S}_{2}\right)=1$, we deduce

$$
\operatorname{dim}\left(\mathbf{k}\left(\widehat{\Sigma} \otimes S_{1}\right)\right) \leq 1 .
$$

As $S_{1}$ is not completely integrable, by Proposition 3.2 we have

$$
\operatorname{rank}\left(\mathbf{k}_{1}\right) \geq 2 \text {. }
$$

By using from (3) through (6) it follows that

$$
\operatorname{rank}\left(\mathbf{k}_{1}\right)=2 \text {. }
$$

Remark 5.3. Example 5.2 shows that it is essential $\operatorname{dim}\left(S_{1} / S_{2}\right)=1$ to obtain $\operatorname{rank}\left(\mathbf{k}_{1}\right)=2$.

The above theorem together with Theorem 5.1 give a direct and intrinsic proof of the next result:

\section{Theorem 5.4.}

$$
\operatorname{dim}\left(S / S_{1}\right)=\operatorname{dim}\left(S_{1} / S_{2}\right)=1 \text { if and only if } \operatorname{rank}(\mathbf{k})=\operatorname{rank}\left(\mathbf{k}_{1}\right)=2 .
$$

Thus, in this case, $S$ and $S_{1}$ have constant class equal to $\operatorname{rank}(S)+2$ and $\operatorname{rank}\left(S_{1}\right)+2$ respectively.

A demonstration using coordinates of the necessary condition of Theorem 5.4 can be seen in $[8$.

It is possible to reformulate Theorem 5.4 in the following terms:

Theorem 5.5. Let $S$ be a totally regular Pfaffian system with derived length $\ell \geq 2$ :

$$
\operatorname{dim}\left(S / S_{2}\right)=2 \text { if and only if } \operatorname{dim}(C / S)=\operatorname{dim}\left(C_{1} / S_{1}\right)=2 .
$$

Notice that we improve the result obtained by R.B. Gardner in [5], in the sense that we do not need to make any assumption about the integrability of $S_{2}$. Aside from this, the proof we give is not in coordinates.

Corollary 5.3. If $S$ is a flag system, with derived length $\ell \geq 2$, then all the derived systems $S_{\mathrm{r}}, 0 \leq \mathrm{r} \leq \ell$, have constant class. The class of $S_{\ell}$ is equal to its rank and the one of $S_{\mathrm{r}}, \mathrm{r}<\ell$, is equal to $\operatorname{rank}\left(S_{\mathrm{r}}\right)+2$.

Reciprocally, if $S$ is a totally regular Pfaffian system with derived length $\ell \geq 2$, such that $\operatorname{rank}\left(\mathbf{k}_{\mathrm{r}}\right)=2$, for every $\mathrm{r}<\ell$, then $S$ is a flag system.

\section{REFERENCES}

[1] R. BRYANT, S. S. CHERN, R. B. GARDNER, H. GOLDSCHMIDT \& P. A. GRIFFITHS, Exterior Differential Systems, MSRI Pub., 18, Springer-Verlag, New York (1991). MR 92h:58007

[2] M. A. CAÑADAS-PINEDO \& C. RUIZ, Differential Invariants of Pfaffian Systems. Equivalence in dimension five, Anales de Física, Monografías, 3 (1996), 71-81. MR 97m:58005

[3] M. A. CAÑADAS-PINEDO \& C. RUIZ, Sternberg's structure function of differential systems in dimension five, Czechoslovak Math. J., 43 (1993), 429-438. MR 94k:58005 
[4] É. CARTAN, Les systèmes de Pfaff à cinq variables et les équations aux dérivées partielles du seconde ordre, Ann. Sc. Normale Sup., 27 (1910), 109-192.

[5] R. B. GARDNER, Invariants of Pfaffian systems, Trans. Amer. Math. Soc., 126 (1967), 514-533. MR 35:2233

[6] R. B. GARDNER \& N. KAMRAN, Normal Forms and Focal Systems for Determined Systems of Two First-Order Partial Differential Equations in the Plane, Indiana Univ. Math. J., 44 (1995), 1127-1162. MR 97i:58006

[7] M. GASPAR, A. KUMPERA \& C. RUIZ, Sur les systèmes de Pfaff en Drapeau, An. Acad. Brasil. Ciênc., 55 (1983), 225-230. MR 85e:58001

[8] A. KUMPERA \& C. RUIZ, Sur l'équivalence locale des Systèmes de Pfaff en Drapeau, Ist. Naz. Alta Mat. Francesco Severi, Rome, 1982. MR 84k:58011

[9] P. J. OLVER, Applications of Lie Groups to Differential Equations, Second Edition, Graduate Texts in Math., 107, Springer-Verlag, New York (1993). MR 94g:58260

[10] S. STERNBERG, Lectures on Differential Geometry, Prentice-Hall, Inc., Englewood Cliffs, N.J. (1964). MR 33:1797

[11] K. YANG, Exterior Differential Systems and Equivalence Problems, Mathematics and its Applications, Kluwer Academic Pub., Dordrecht (1992). MR 93m:58005

Departamento Álgebra, Geometría y Topología, Fac. Ciencias, Universidad de Málaga, Campus de Teatinos, Apdo. 59, 29080 - Málaga, Spain

E-mail address: pinedo@uma.es

Departamento Geometría y Topología, Universidad de Granada, 18071 - Granada, SPAIN

E-mail address: ruiz@ugr.es 